\title{
Lesão renal aguda em pacientes diagnosticados com COVID-19 em uma UTI do
}

\section{sudoeste goiano}

\author{
Acute kidney injury in patients diagnosed with COVID-19 in an ICU in southwestern Goiás \\ Lesión renal aguda en pacientes diagnosticados de COVID-19 en una UCI del suroeste de Goiás
}

Recebido: 07/12/2021 | Revisado: 12/12/2021 | Aceito: 17/12/2021 | Publicado: 22/12/2021

\author{
Lalleska Fernandes Carvalho \\ ORCID: https://orcid.org/0000-0002-9698-8783 \\ Faculdade Morgana Potrich, Brasil \\ E-mail: lalleska.carvalhof@gmail.com \\ Tatyane Christina Gomes Queiroz de Paula \\ ORCID: https://orcid.org/0000-0003-0153-084X \\ Faculdade Morgana Potrich, Brasil \\ E-mail: tatyapaula94@gmail.com \\ Valeria Silva Peixoto \\ ORCID: https://orcid.org/0000-0001-8992-3669 \\ Faculdade Morgana Potrich, Brasil \\ E-mail: valeriasilva@fampfaculdade.com.br
}

\begin{abstract}
Resumo
Das apresentações clínicas da COVID-19, a insuficiência respiratória demanda maior destaque, porém o acometimento renal é descrito como um quadro clínico relevante. A Lesão Renal Aguda (LRA) é definida como a perda abrupta da função renal e tem sido associada a taxas elevadas de mortalidade e a um prognóstico renal desfavorável em pacientes críticos com a infecção. Objetivo: Analisar a incidência da insuficiência renal aguda secundária a COVID-19, em uma Unidade de Terapia Intensiva (UTI). Método: Pesquisa qualitativa, quantitativa e retrospectivo documental em prontuários de pacientes de uma UTI do Sudoeste Goiano com diagnóstico positivo para COVID-19, internos durante 1 de setembro de 2020 a 30 de abril de 2021. Para estratificação da LRA foram considerados os critérios de KDIGO. Resultados: Dos 212 pacientes, 60 evoluíram para LRA, com predominância de $80 \%$ em estágio 3. A idade média foi de 62,6 e maior incidência no sexo masculino $51,67 \%$. O tempo médio de internação hospitalar foi de 11,6 dias. As comorbidades de maior prevalência foram: Hipertensão arterial: 53,33 \% e Diabetes Melitus: $31,67 \%$. Em relação às drogas nefrotóxicas, $78,33 \%$ são vasodilatadoras. Das complicações clínicas: 88,33\% necessitaram de suporte ventilatório invasivo e $80 \%$ de Hemodiálise, já 93,33 \% evoluíram para óbito. Conclusão: Conclui-se que houve alta incidência de LRA, possuindo forte influência para mortalidade, principalmente associado a fatores de risco como idade elevada, comorbidades e o manejo clínico como o suporte ventilatório, necessidade de hemodiálise e o uso de drogas vasoativas.
\end{abstract}

Palavras-chave: SARS-CoV-2; Lesão renal aguda; Unidade de Tratamento Intensivo.

\begin{abstract}
Respiratory failure is, from the clinical presentations of COVID-19, the one that demands greater prominence, but kidney involvement is described as a relevant clinical picture. Acute Kidney Injury (AKI) is defined as the abrupt loss of kidney function and has been associated with high mortality rates and a poor renal prognosis in critically ill patients with the infection. Objective: Analyze the incidence of acute renal failure secondary to COVID-19 in an Intensive Care Unit (ICU). Method: Qualitative, quantitative and retrospective documentary research in medical records of patients from an ICU in Southwest Goiás with a positive diagnosis for COVID-19, interned during September 1, 2020 to April 30, 2021. For AKI stratification, the criteria of AKI were considered. KDIGO. Results: From the 212 patients, 60 progressed to AKI, with a predominance of $80 \%$ in stage 3. The average age was 62.6 and the highest incidence in males was $51.67 \%$. The average hospital stay was 11.6 days. The most prevalent comorbidities were: Hypertension: $53.33 \%$ and Diabetes Melitus: $31.67 \%$. Regarding nephrotoxic drugs, $78.33 \%$ are vasodilators. The clinical complications: $88.33 \%$ required invasive ventilatory support and, $80 \%$ needed hemodialysis, whereas $93.33 \%$ progressed to death. Conclusion: It is concluded that there was a high incidence of AKI, with a strong influence on mortality, mainly associated with risk factors such as older age, comorbidities and clinical management such as ventilatory support, need for hemodialysis and the use of vasoactive drugs.
\end{abstract}

Keywords: SARS-CoV-2; Acute kidney injury; Intensive Care Unit. 


\begin{abstract}
Resumen
De las presentaciones clínicas de COVID-19, la insuficiencia respiratoria exige un mayor protagonismo, pero la afectación renal se describe como un cuadro clínico relevante. La lesión renal aguda (LRA) se define como la pérdida abrupta de la función renal y se ha asociado con altas tasas de mortalidad y un mal pronóstico renal en pacientes despiertos con la infección. Objetivo: Analizar la incidencia de insuficiencia renal aguda secundaria a COVID-19 en una Unidad de Cuidados Intensivos (UCI). Método: Investigación documental cualitativa, cuantitativa y retrospectiva en historias clínicas de pacientes de una UCI en el suroeste de Goiás con diagnóstico positivo para COVID-19, internados durante el 1 de septiembre de 2020 al 30 de abril de 2021. Para la estratificación de LRA, los criterios de LRA fueron considerado KDIGO. Resultados: De los 212 pacientes, 60 progresaron a LRA, con predominio del $80 \%$ en el estadio 3. La edad media fue de 62,6 años y la mayor incidencia en varones fue de 51,67\%. La estancia hospitalaria media fue de 11,6 días. Las comorbilidades más prevalentes fueron: Hipertensión: 53,33\% y Diabetes Melitus: $31,67 \%$. En cuanto a los fármacos nefrotóxicos, el 78,33\% son vasodilatadores. De las complicaciones clínicas: el 88,33\% requirió soporte ventilatorio invasivo y el 80\% necesitó hemodiálisis, mientras que el 93,33\% progresó hasta la muerte. Conclusión: Se concluye que hubo una alta incidencia de LRA, con fuerte influencia en la mortalidad, principalmente asociada a factores de riesgo como edad avanzada, comorbilidades y manejo clínico como soporte ventilatorio, necesidad de hemodiálisis y uso de fármacos vasoactivos

Palabras clave: SARS-CoV-2; Lesión renal aguda; Unidad de Cuidados Intensivo.
\end{abstract}

\title{
1. Introdução
}

Em dezembro de 2019, foi descrito um surto de pneumonia de origem até então desconhecida em Wuhan, na China. O agente etiológico se tratava de uma infecção viral causada pelo novo betacoronavírus, sendo mais tarde nomeado de SARSCoV-2, responsável pela síndrome respiratória aguda grave. Em 30 de janeiro, a Organização Mundial de Saúde (OMS) emitiu o mais alto nível de alerta, configurando em Emergência de Saúde Pública de Importância Internacional (ESPII). A COVID-19 tomou rapidamente proporções mundiais, sendo declarada pandemia pela OMS em 11 de março de 2020.

A pandemia causada pelo novo coronavírus evidenciou uma grave crise emergencial e apontou uma fragilidade mundial em saúde pública, implicando em uma constante análise e alerta da comunidade científica na identificação de complicações secundárias à infecção. A COVID-19 é uma doença respiratória, porém pode apresentar manifestações extrapulmonares, como complicações neurológicas, cardiovasculares e renais, caracterizando-a como uma doença multissistêmica (Anazco et al., 2021).

Segundo Kunutsor e Laukkanen (2020), das manifestações extrapulmonares, o dano renal é descrito como uma complicação da COVID-19 grave, da qual frequentemente contribui para Lesão Renal Aguda (LRA). Sabe-se que a entrada do vírus nas células é mediada pela interação da Glicoproteína S nos receptores da enzima conversora de angiotensina 2 (ECA2), e proteases trans membrana tipo 2 (TMPRSS2). O tecido renal possui alta expressividade do gene da ECA2, principalmente nos túbulos renais, e em menor quantidade nos podócitos. A LRA pode ser desencadeado por: desequilíbrio da angiotensina, estimulação de respostas imunes, fatores de coagulação e tempestade de citocinas (De Matos, et. al. 2021)

Dentre as hipóteses do acometimento renal, é exemplificado por dois mecanismos, sendo de ação direta nos receptores da Enzima Conversora de Angiotensina (ECA2), a qual é altamente expressa nos rins, causando lesão renal proveniente por ocitocinas inflamatórias resultantes da infecção e também pela trombose microvascular. Já nos mecanismos indiretos, a maioria dos pacientes graves apresentam uma alta incidência de doenças pré-existentes, como hipertensão arterial sistêmica e diabetes mellitus, que já são altamente voltadas para a ocorrência de lesão renal (Gabarre, et al. 2020). Ainda não está totalmente elucidado até que ponto a lesão renal é decorrente da injúria direta no órgão, crosstalk de órgãos, uso de suporte de ventilação mecânica, drogas nefrotóxicas, ou se é mediada, principalmente, pela tempestade de citocinas.

O objetivo do estudo foi o de compreender e avaliar a incidência da lesão renal secundária a COVID-19, como sendo de fundamental importância para o entendimento das complicações da doença, contribuindo para o manejo terapêutico.

Em decorrência do exposto, respondeu-se a seguinte questão: Qual a incidência de Lesão Renal Aguda em pacientes com infecção causada pelo SARS-CoV-2 internados em uma UTI no Sudoeste Goiano? Sendo assim, esse trabalho se 
justificou da necessidade de compreender os mecanismos da doença no comprometimento renal e elucidar um melhor entendimento acerca das complicações clínicas implicadas pela doença do novo Coronavírus.

\section{Metodologia}

A pesquisa possui caráter qualitativo, quantitativo e retrospectivo documental (Lemos, 2018). Aprovado pelo Comitê de Ética da Faculdades Integradas de Santa Fé do Sul - FISA/FUNEC com CAAE: 50131921.6.0000.5428, com o objetivo de análise da incidência da insuficiência renal aguda secundária por Covid-19, em pacientes criticamente enfermos em uma UTI.

O estudo foi desenvolvido em uma Unidade de Terapia Intensiva de um hospital municipal do Sudoeste Goiano. É uma unidade de campanha para o enfrentamento da pandemia, possuindo classificação de UTI - Covid, com total de 12 leitos. Para a realização do estudo, foi efetuado um levantamento de dados através de livros de registros e prontuários eletrônicos dentro da própria unidade hospitalar. Foi elaborado um check-list com os dados definidos pelo questionário, a fim de direcionar a investigação compreendida no período de $1^{\circ}$ de setembro de 2020 a 30 de abril de 2021 . A disposição dos dados e a análise estatística ocorreram através do Microsoft Excel.

A amostra foi estabelecida conforme a aplicação dos critérios de inclusão: idade superior ou igual a 18 anos, com teste positivo por reação em cadeia da polimerase (RT-PCR) em swab de orofaringe e permanência hospitalar maior que 48 horas, no tempo proposto pela pesquisa. Adotados como critérios de exclusão, pacientes com idade inferior a 18 anos, estadia na unidade menor do que 48 horas, re-internação durante o tempo proposto, histórico de disfunções renais anterior à infecção por SARS-Cov-2 (paciente renal crônico, dependente de diálise ou outro mecanismo de terapia renal substitutiva) e que não tenha informação referente ao débito urinário, conforme a (Figura 1).

Figura 1 Fluxograma elegibilidade da pesquisa.

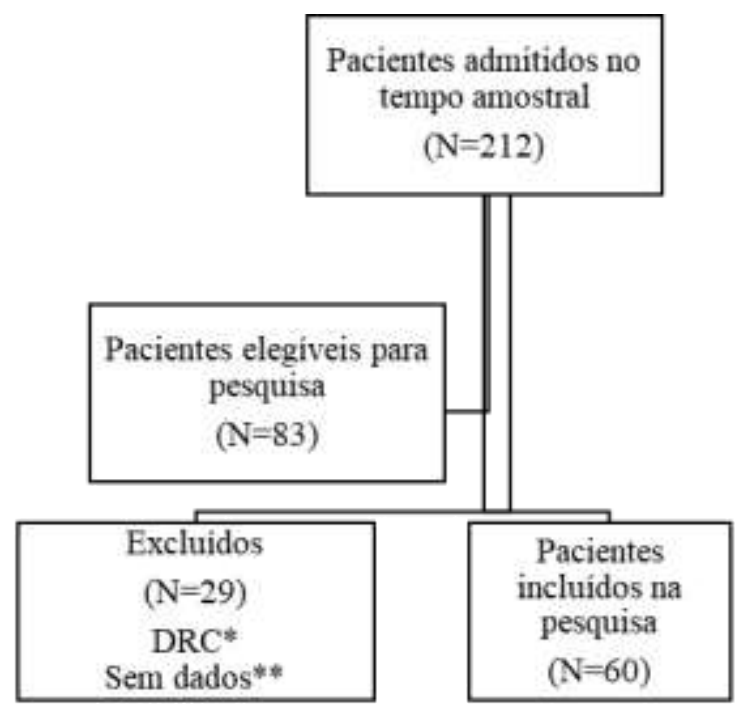

* Doença renal crônica. ** Sem dados de creatinina ou débito urinário. Fonte: Autoras.

\section{Resultados e Discussão}

A partir da análise de dados, foi perceptível a maior incidência do sexo masculino $(51,67 \%)$ em relação à $(48,33 \%)$ do sexo feminino, conforme a (Tabela 1). A predominância de pacientes do sexo é semelhante aos resultados de outros estudos, como o de Lopes, et. al. (2018), que objetivou descrever o perfil dos pacientes com evolução para insuficiência renal em uma UTI de um hospital universitário no interior do Paraná, Brasil, apresentando maior incidência do gênero masculino 
(55,84\%). Para o autor Dantas, et. al. (2021), a prevalência do sexo masculino é evidenciada pela negligência do sexo com sua saúde, pela ausência ou baixa procura dos serviços de saúde.

No que se refere à faixa etária de predominância, resultou maior incidência entre os 60 a 69 anos com (40\%), seguido da faixa de $70>\operatorname{com}(28,33 \%)$ e com idade média de 62,2 anos. A respeito da permanência hospitalar, a maior incidência foi de 8 a 15 dias com (40\%), seguido de 2 a 7 dias com segunda maior faixa (36,67\%), com média de dias de 11,6. Um estudo realizado na China $(\mathrm{N}=221)$ evidenciou que a idade média dos pacientes críticos $(\mathrm{N}=55)$ era predominantemente alta $(62,0$ anos), fato condizente com maior possibilidade de comorbidades, o que propicia o desenvolvimento de complicações clinicas e maior estadia hospitalar (Zhang, et. al. 2020).

Tabela 1: Variáveis socioepidemiológicas, Mineiros, 2021.

\begin{tabular}{ccc}
\hline Gênero & Fa & Fr \% \\
\hline Masculino & 31 & $51,67 \%$ \\
Feminino & 29 & $48,33 \%$ \\
\hline Faixa etária & Fa & Fr \% \\
\hline 29 a 49 & 10 & $16,67 \%$ \\
50 a 59 & 9 & $15 \%$ \\
60 a 69 & 24 & $40 \%$ \\
$70>$ & 17 & $28,33 \%$ \\
\hline Tempo de permanência & Fa & Fr \% \\
\hline 2 a 7 & 22 & $36,67 \%$ \\
8 a 15 & 24 & $40 \%$ \\
18 a 20 & 6 & $10 \%$ \\
21 a 37 & 8 & $13,33 \%$ \\
\hline
\end{tabular}

Fonte: Dados dos autores.

A respeito das comorbidades de prevalência nos pacientes, obtiveram-se os seguintes resultados: Doenças cardiovasculares: (6,67\%), Diabetes Mellitus: (31,67\%), Hipertensão arterial sistêmica: (53,33\%) e doenças respiratórias: $(1,67 \%$ ) (Tabela 2). De acordo com Almeida, et al. (2020) as informações corroboram em relação às comorbidades clínicas na covid-19 que atuam como fatores agravantes para evolução da doença, uma vez que sobrecarregam o organismo, impossibilitando a eficácia completa do sistema imune no combate da infecção viral.

Um estudo de Silva et al. (2021) analisou a incidência e fatores relacionados com a injúria renal aguda em pacientes críticos, em uma amostra de $(\mathrm{N}=30)$ pacientes, evidenciou que 63,40\% dispunha de hipertensão arterial sistêmica (HAS), e 46,60\% possuíam Diabetes Mellitus (DM). O autor ressalta ainda que são as comorbidades de caráter mais comuns em pacientes críticos com evolução para injuria renal aguda. Segundo De Souza, et al. (2020), os portadores de DM e HAS tendem a desenvolver a forma grave da doença devido a alto nível de ECA2, visto que ambas patologias, quando tratadas com inibidores da ECA e bloqueadores dos receptores da angiotensina (BRA), resulta em elevadas taxas de ECA2 por regulação positiva, o que facilitaria a infecção. 
Tabela 2: Presença de comorbidades, Mineiros, 2021.

\begin{tabular}{lcc}
\hline $\begin{array}{l}\text { Hipertensão arterial } \\
\text { sistêmica }\end{array}$ & Fa & Fr \% \\
\hline Sim & 32 & $53,33 \%$ \\
Não & 28 & $46,67 \%$ \\
\hline Diabetes Mellitus & Fa & Fr \% \\
\hline Sim & 19 & $31,67 \%$ \\
Não & 41 & $68,33 \%$ \\
\hline Doenças cardiovasculares & Fr \% & $6,67 \%$ \\
\hline Sim & 4 & $93,33 \%$ \\
Não & 56 & Fr \% \\
\hline Doenças respiratórias & Fa & $1,67 \%$ \\
\hline Sim & 1 & $98,33 \%$ \\
\hline Não & 59 & \\
\hline
\end{tabular}

Fonte: Dados dos autores.

Em relação à incidência de LRA, obtiveram-se os seguintes resultados: Lesão renal estágio um: 11,67\%, lesão renal estágio dois: 8,33\% e lesão renal em estágio três: $80 \%$ (Tabela 3). Nos países mais atingidos pela infecção (China, Itália e Estados Unidos), a injúria renal manifestou em cerca de $20 \%$ dos pacientes críticos e naqueles com evolução para óbito. Salienta-se ainda um prognóstico renal desfavorável, com progressão da LRA para cronicidade (Lima, et. al. 2021).

A respeito do volume de diurese: 31,67\% manifestaram anúria, já 28,33\% oligúria e os outros $40 \%$ volume normal. (Tabela 3). A maioria dos pacientes apresentaram balanço hídrico positivo. Para a estratificação dos estágios 1 e 2 , foi considerado valor da creatinina ou debito urinário, considerando os critérios da KDIGO, enquanto que os pacientes que evoluíram para terapia renal substitutiva foram classificados automaticamente em estágio 3, uma vez que independe dos resultados das escórias renais quando há evolução para hemodiálise.

A infecção pelo SARS-CoV-2 possui uma patogênese de lesão renal ainda não muito bem compreendida, nem definida. O dano renal causado pela ação viral é principalmente tubular, com prejuízos na filtração glomerular e apresentação de altos níveis de ureia e creatinina. Com isso, há um comprometimento geral da função renal, ocasionando um quadro agudo de lesão renal (DUARTE, et.al. 2020). Essa patogênese pode ser explicada em três aspectos: (I) injúria induzida por citocinas, (II) crosstalk de órgãos e (III) manifestações sistêmicas. Tais mecanismos citados funcionam interligados (Poloni, et. al. 2020).

Chagas, et. al. (2021) indicaram a necrose tubular aguda (NTA) isquêmica como a causa fundamental para ocorrência de LRA na COVID-19, decorrendo em 66\%, resultante principalmente de hipotensão e depleção de volume. Corrobora ainda que a nefrotoxidade de contraste de exames de imagem, rabdomiólise, pouca ingesta hídrica, hiperexia e disfunção de múltiplos órgãos, choque, acidose metabólica e hipercalemia são manifestações comuns em pacientes com COVID-19 e, consequentemente, contribuem para injuria no tecido renal. Em análises de pós-morte foi identificada a presença do vírus em células renais e em sedimentos urinários, relacionada principalmente com a nefrite em túbulo intersticial, evidenciando ainda mais a possibilidade do rim como órgão alvo do vírus, acometido por lesão direta (Rodrigues, et al. 2020). 
Tabela 3: Informações gerais referente Lesão renal Aguda, Mineiros, 2021.

\begin{tabular}{|c|c|c|}
\hline $\begin{array}{l}\text { Lesão renal aguda } \\
\text { KDIGO }\end{array}$ & $\mathbf{F a}$ & Fr \% \\
\hline Estágio 1 & 7 & $11,67 \%$ \\
\hline Estágio 2 & 5 & $8,33 \%$ \\
\hline Estágio 3 & 48 & $80,00 \%$ \\
\hline Volume de diurese & $\mathbf{F a}$ & Fr \% \\
\hline Oligúria & 17 & $28,33 \%$ \\
\hline Volume normal & 24 & $40 \%$ \\
\hline
\end{tabular}

Fonte: Dados dos autores.

Em virtude das complicações clínicas impostas pela infecção do SARS-CoV-2, 88,33\% estavam em uso de ventilação mecânica invasiva, 80 \% necessitaram de hemodiálise e 93,33 \% evoluíram para óbito (Tabela 4).

Quanto à necessidade de ventilação mecânica invasiva (VMI) é um fator agravante de forma significativa para a ocorrência de desarranjos na função renal, devido à estreita relação da pressão intratorácica resultante da ventilação e dos pulmões com complacência reduzida, o que possibilita diminuição do débito cardíaco, levando a uma hipoperfusão renal (Panitchote, et al. 2021). Segundo Pecly et. al. (2021), a alta pressão não só causa lesão a nível pulmonar, como contribui também para uma inflamação sistêmica. Além disso, valores altos da pressão positiva no final da expiração (PEEP) estão associados à hipercitocinemia e à LRA. O aumento da inflamação causa apoptose de células pulmonares, mais precisamente alveolar, ocasionando a elevação da permeabilidade vascular, definindo um eixo pulmão-rim.

Corroborando com os resultados obtidos, uma pesquisa de coorte prospectiva em uma UTI do hospital no interior amazônico analisou $(\mathrm{N}=79)$ pacientes, objetivou verificar a relação da Ventilação mecânica e da pressão positiva com a predisposição de ocorrer LRA. A análise de 79 pacientes evidenciou que 59,5\% necessitaram do suporte ventilatório invasivo (Cunha, et. al. 2019). Outro estudo, com objetivos semelhante, corrobora que VMI é umas das intervenções mais utilizadas em pacientes críticos, porém uma condição contributiva para a injuria renal. Ressalta ainda que paciente em PEEP > $5 \mathrm{~cm} H 2 \mathrm{O}$ são mais propensos em desenvolver LRA (Silva, et. al. 2021).

No que se refere à terapia renal substitutiva, a hemodiálise apresenta umas das condutas mais frequentes em pacientes críticos. Um estudo realizado na Inglaterra com $(\mathrm{N}=52)$ pacientes evidenciou que 17 \% evoluíram para necessidade de hemodiálise. Ressalta ainda a relevância de profissionais competentes e qualificados devido às práticas de procedimentos invasivos, com destaque para a atuação do enfermeiro (De lima, et. al. 2021). Ainda, Silva, et. al. (2018) corrobora que as principais complicações durante as sessões de hemodiálise em pacientes críticos, frequentemente: hipotensão, arritmias, hipoglicemia, coagulação no circuito e hipotermia.

Em relação aos óbitos (Tabela 4), os dados obtidos sobre a incidência de mortalidade nos sexos totalizaram de 53,57 $\%(\mathrm{~N}=30)$ do sexo masculino sobre 46,43\% $(\mathrm{N}=26)$ do sexo feminino. Tais dados conferem com os resultados anteriores de maior prevalência do gênero masculino na internação e com o fato de ser um grupo com maior susceptibilidade em fatores de riscos. De acordo com Costa, et. al. (2021), em sua pesquisa de coorte $(\mathrm{N}=102)$ pacientes, a amostra sem lesão renal apresentou 8,9\% de óbitos, enquanto que os pacientes que apresentaram LRA 33,3\% faleceram.

A pesquisa de Andrade, et.al. (2021) objetivou analisar o perfil de mortalidade dos pacientes diagnosticados com Covid-19, internos em uma UTI de um hospital público na Região Sul da Amazônia, Brasil. Sendo analisado total de (N=151) prontuários, resultou que a mortalidade da amostra totalizou cerca 54,3\% e demostrou conclusões semelhantes em relação aos sexos, contendo maior índice de mortalidade no sexo masculino 64,04\%, comparado ao sexo feminino 40,32\%. Outro estudo com mesmo objetivo da pesquisa anterior, analisou $(\mathrm{N}=50)$ prontuários de pacientes que evoluíram para óbito e evidenciou que $62 \%$ eram do sexo masculino e $19 \%$ do sexo feminino (Santos, et. al. 2021). 
Tabela 4: Complicações da infecção, Mineiros, 2021.

\begin{tabular}{lcc}
\hline $\begin{array}{l}\text { Ventilação mecânica } \\
\text { invasiva }\end{array}$ & Fa & Fr \% \\
\hline Sim & 53 & $88,33 \%$ \\
Não & 7 & $11,67 \%$ \\
\hline Hemodiálise & Fa $\%$ & $80,00 \%$ \\
\hline Sim & 48 & $20,00 \%$ \\
Não & 12 & Fr \% \\
\hline Óbito & Fa & $93,33 \%$ \\
Sim & 56 & $6,67 \%$ \\
Não & 4 & \\
\hline
\end{tabular}

Fonte: Dados dos autores.

Em relação ao uso de drogas nefrotóxicas, 78,33 \% estavam em uso de drogas vasoativas e 81,67 \% em uso de antibióticos, já 66,67\% utilizaram anticoagulantes (Tabela 5).

O uso de drogas vasoativas apresenta relação significante com o desenvolvimento da injúria renal, constituindo fator intensamente negativo. Portanto é reconhecido, que o uso de drogas vasoativas constitui importante fator de risco para mortalidade em terapia intensiva, principalmente devido à instabilidade hemodinâmica dos pacientes, e em função do mecanismo de vasoconstrição (Da Rocha et al. 2017).

Em relação aos eventos tromboembólicos na Covid-19, evidenciou que $20 \%$ a $50 \%$ dos pacientes que necessitam de internação apresentam o aumento de biomarcadores específicos, como D-dímero e tempo de protrombina. Corrobora ainda que cerca de $69 \%$ dos pacientes com a infecção evoluíram para tromboembolismo venoso e $71 \%$ dos óbitos eram compatíveis com distúrbios de coagulação, fato que demostra o risco elevados desses pacientes em mortalidade intra-hospitalar (Do Vale, et. al. 2021).

Em função do uso de drogas, tanto a função tubular e glomerular é acometida em resposta a lesão renal, o que consequentemente possibilita a ocorrência de um quadro agudo ou até mesmo crônico. Ainda possui certas limitações de estudos referente a injuria causada por medicamentos, principalmente a curtos prazos. Em uma análise de (N=95) pacientes, verificou que o risco relativo $(\mathrm{RR})$ de apresentar injuria renal em uso de antibióticos, obteve resultado de $-\mathrm{RR}=3,0321$, dentro de um intervalo $95 \%,(1,6369$ a 5,6165). Isto é, o risco de desenvolver a LRA é três vezes maior quando comparado a um paciente que não fez uso de antibioticoterapia (Lima, et. al. 2018).

Tabela 5: Drogas nefrotóxicas, Mineiros, 2021.

\begin{tabular}{lll}
\hline Drogas vasoativas & Fa & Fr \% \\
\hline Sim & 47 & $78,33 \%$ \\
Não & 13 & $21,67 \%$ \\
\hline Anticoagulantes & Fa & Fr \% \\
\hline Sim & 40 & $66,67 \%$ \\
Não & 20 & $33,33 \%$ \\
\hline Antibioticoterapia & Fa & Fr \% \\
\hline Sim & 49 & $81,67 \%$ \\
Não & 11 & $18,33 \%$ \\
\hline
\end{tabular}

Fonte: Dados dos autores.

\section{Conclusão}

É sabido que a COVID-19 não é uma doença restrita apenas ao dano no aparelho respiratório, como também apresenta 
o acometimento de outros órgãos. A lesão renal aguda é uma condição inerente às complicações da doença em pacientes internados nessa UTI, essencialmente quando relacionada a fatores de risco como a idade avançada e presença de comorbidades, e o tempo de estadia no setor o que influencia principalmente no manejo clinico, como a necessidade de ventilação mecânica invasiva e o uso de drogas vasoativas devido a instabilidade hemodinâmica desses pacientes.

Desse modo, se destaca também a importância da capacitação e continuidade de formação dos profissionais que lidam diretamente com os pacientes dentro de uma UTI. Apesar das constantes buscas e estudos para elucidar os processos patológicos da infecção, entende-se que por se tratar de um assunto um tanto ainda recente, as fontes cientificas sobre a relação da COVID-19 e a lesão renal são escassas e se tratando de hipóteses. Portanto, torna-se evidente a necessidade de mais estudos a respeito da temática, principalmente quanto a relação de doenças secundárias a infecção pelo Sars-cov-2, o que possibilita compreender o curso clínico da doença, uma vez que é fundamental para estratégias de enfrentamento neste cenário.

\section{Referências}

Añazco, P. H., Balta, F. M., \& Córdova-Cueva, L. (2021). Bilateral renal infarction in a patient with severe COVID-19 infection. Brazilian Journal of Nephrology, 43 (1) 127-131.

Andrade, G. D., Kundsin, A., Dias, S. A., \& Santos, G. T. (2021). Perfil de mortalidade associado à pandemia de infecção por SARs-CoV-2 em um Hospital Público da Região Sul da Amazônia Ocidental. Research, Society and Development, 10 (13), e288101321359-e288101321359.

Chagas, G. C. L., Rangel, A. R., Noronha, L. M., Meneses, G. C., Martins, A. M. C., \& Daher, E. D. F. (2021). COVID-19 e os rins: uma revisão narrativa. Revista Brasileira de Saúde Materno Infantil, 21 (2) 373-381.

Costa, R. L. D., Sória, T. C., Salles, E. F., Gerecht, A. V., Corvisier, M. F., Menezes, M. A. D. M., \& Simvoulidis, L. F. N. (2021). Lesão renal aguda em pacientes com Covid-19 de uma UTI no Brasil: incidência, preditores e mortalidade hospitalar. Brazilian Journal of Nephrology, 43 (3) 349 -358.

Cunha, N. V. A., de Sousa Santana, B., da Paixão Duarte, T. T., de Lima, W. L., Nava, L. F., \& da Silva Magro, M. C. (2019). Pressão positiva na ventilação mecânica invasiva e implicações renais em pacientes críticos. Revista de Enfermagem do Centro-Oeste Mineiro, 9, 3505.

Da Rocha Guedes, J., da Silva, E. S., do Nascimento Carvalho, I. L., \& de Oliveira, M. D. (2017). Incidência e fatores predisponentes de insuficiência renal aguda em unidade de terapia intensiva. Cogitare Enfermagem, 22 (2), e49035

Dantas, L. A. L., Vieira, A. N., Oliveira, L. C. de, Araújo, M. E. da S., \& Maximiano, L. C. de S. (2021). Fatores de risco para Lesão Renal Aguda em Unidade de Terapia Intensiva. Research, Society and Development, 10 (6), e32210615700.

De Almeida, J. O., de Oliveira, V. R. T., Avelar, J. L. S., Moita, B. S., \& Lima, L. M. (2020). COVID-19: Fisiopatologia e Alvos para Intervenção Terapêutica. Rev. Virtual Quim. ISSN, 12 (6), 1464-1497.

de Lima Neves, J., Schwartz, E., de Lima Spagnolo, L. M., Ávila, J. P., de Oliveira, N. P., \& Lise, F. (2021). Complicações associadas a COVID-19 e as principais necessidades humanas básicas afetadas. Enfermagem Brasil, 20 (1), 94-108.

de Souza, L. C., da Silva, T. O., da Silva Pinheiro, A. R., \& dos Santos, F. D. S. (2021). SARS-CoV, MERS-CoV e SARS-CoV-2: uma revisão narrativa dos principais Coronavírus do século. Brazilian Journal of Health Review, 4 (1), 1419-1439.

Do Vale, V. A. L., Martins, J. B., Grossi, J. E. Q., Durães, P. A. A., Campos, R. M. H., Ribeiro, T. M., ... \& da Silva Reis, F. J. (2021). Probabilidade de eventos Tromboembólicos em pacientes com COVID-19: patogênese e profilaxia. Revista Eletrônica Acervo Saúde, 13 (8), e8433.

Duarte, P. M. D. A., Bastos, F. A. G., Duarte, J. V. A., Duarte, B. A., Duarte, I. A., Lemes, R. P. G., \& Duarte, F. B. (2020). Renal changes in COVID-19 infection. Revista da Associação Médica Brasileira, 66 (10) 1335-1337.

Gabarre, P., Dumas, G., Dupont, T., Darmon, M., Azoulay, E., \& Zafrani, L. (2020). Acute kidney injury in critically ill patients with COVID-19. Intensive care medicine, 46 (7), 1339-1348.

Kunutsor, S. K., \& Laukkanen, J. A. (2020). Renal complications in COVID-19: a systematic review and meta-analysis. Annals of medicine, 52 (7), $345-353$. Lemos, G. C. (2018). Metodologia Científica: a pesquisa qualitativa nas visões de Lüdke e André. Revista Eletrônica Científica Ensino Interdisciplinar. 4 (12), $531-541$.

Lima, R. Q. (2018). Lesão renal aguda pós uso de antibióticos. Revista da Faculdade de Medicina de Teresópolis, 2 (1), $172-185$.

Lopes, D., da Silva Schran, L., de Oliveira, J. L. C., Oliveira, R. B. S. R., \& Fernandes, L. M. (2018). Fatores de risco/causais para insuficiência renal aguda em adultos internados em terapia intensiva. Enfermagem Brasil, 17 (4), 336-345.

Matos, A. C. G. de ., Andrade, T. V. de ., Noronha, N. C. M. ., Lima, T. B. ., Nascimento, C. I. S. do ., Santos, A. C. M., Santana, V. S. de J., Maynard, L. G., \& Jesus, C. V. F. de. (2021). Lesão renal aguda na COVID-19 e abordagem multiprofissional. Research, Society and Development, 10 (3), e53310313436.

Panitchote, A., Mehkri, O., Hastings, A., Hanane, T., Demirjian, S., Torbic, H., ... \& Duggal, A. (2019). Factors associated with acute kidney injury in acute respiratory distress syndrome. Annals of intensive care, 9 (1), 1-10. 
Research, Society and Development, v. 10, n. 17, e157101724492, 2021

(CC BY 4.0) | ISSN 2525-3409 | DOI: http://dx.doi.org/10.33448/rsd-v10i17.24492

Pecly, I. M. D., Azevedo, R. B., Muxfeldt, E. S., Botelho, B. G., Albuquerque, G. G., Diniz, P. H. P., Silva, R., \& Rodrigues, C. I. S. (2021). A review of Covid-19 and acute kidney injury. from pathophysiology to clinical results. Braz. J. Nephrol., 00 (00), 00-00.

Poloni, J. A. (2020). Insuficiência renal aguda em pacientes com COVID-19. A Tempestade do Coronavírus, 52 (2), 160-167.

Proetti, S. (2018). As pesquisas qualitativa e quantitativa como métodos de investigação científica: Um estudo comparativo e objetivo. Revista Lumen-ISSN: 2447-8717, 2(4).

Rodrigues, C. M. B., da Costa, N. B., Vieira, V. R., Gabriel, E. A., \& Gabriel, S. A. (2020). COVID-19: sistema renal e cardíaco. ULAKES JOURNAL OF MEDICINE, 1 .

Santos, P. S. A., Mateus, S. R. M., de Oliveira Silva, M. F., de Souza Figueiredo, P. T., \& Campolino, R. G. (2021). Perfil epidemiológico da mortalidade de pacientes internados por Covid-19 na unidade de terapia intensiva de um hospital universitário. Brazilian Journal of Development, 7 (5), $45981-45992$.

Silva, A. F. S., Magalhães, D. M., Rocha, P. R. S., \& da Silva, R. F. (2018). Principais complicações apresentadas durante a hemodiálise em pacientes críticos e propostas de intervenções de enfermagem. Revista de Enfermagem do Centro-Oeste Mineiro, 8 (0) 1-9

Silva, J. B. da, Melo, C. A. de S., \& Barros, T. G. (2021). Injúria renal aguda na Unidade de Terapia Intensiva em um hospital do interior amazônico. Research, Society and Development, 10 (10), e447101019178.

Zhang, G., Hu, C., Luo, L., Fang, F., Chen, Y., Li, J., \& Pan, H. (2020). Clinical features and short-term outcomes of 221 patients with COVID-19 in Wuhan, China. Journal of Clinical Virology, 127, 104364. 